\title{
Use of Caries Assessment Spectrum Treatment (CAST) Severity Score in an Adult Population
}

\section{Castro Ana Luiza Sarno ${ }^{1 *}$, Leal Soraya Coelho ${ }^{2}$, Vianna Maria Isabel Pereira $^{3}$ and Mendes Carlos Maurício Cardeal ${ }^{4}$}

${ }^{1}$ Department of Health, State University of Feira de Santana, Feira de Santana, Bahia, Brazil

${ }^{2}$ Department of Dentistry, School of Health Sciences, University of Brasília, Brasília, Brazil

${ }^{3}$ Department of Public Oral Health, School of Dentistry, Federal University of Bahia, Salvador, Bahia, Brazil

${ }^{4}$ Postgraduate Studies in Interactive Processes of Organs and Systems, Health

Science Institute, Federal University of Bahia, Salvador, Bahia, Brazil

*Corresponding Author: Castro Ana Luiza Sarno, Department of Health, State

University of Feira de Santana, Feira de Santana, Bahia, Brazil.
Received: July 14, 2021

Published: August 12, 2021

(C) All rights are reserved by Castro Ana Luiza

Sarno., et al.

\section{Abstract}

Background: The Caries Assessment Spectrum Treatment (CAST) severity score has been tested in children, but not yet in adults. The objective of the study is to evaluate the applicability of the CAST severity score in grouping adult citizens according to their caries load.

Methods: This descriptive and cross-sectional study used the caries severity calculation formula F1 (tested previously in children) in 260 students, employees and dependents of the Medical, Dental and Social Service at the State University of Bahia, Salvador, Brazil. F1 was applied in two different ways: including CAST code 8- missing teeth due to caries (F1) or not (F1a).

Results: The population distribution according to the CAST severity score using F1 was: mild (34.6\%), moderate (31.2\%), and severe (34.2\%). And applying F1a was: mild (35.0\%), moderate (33.5\%) and severe (31.5\%).

Conclusion: The maximum CAST score per subject and the CAST severity score proved to be good tools to assess the severity and prevalence of caries in the study group; however, excluding the missing component resulted in a better classification of individuals and made the values less heterogeneous.

Keywords: Epidemiology; CAST; Dental Caries; Severity of Illness Index; Oral Health Status

\section{Abbreviation}

CAST: Caries Assessment Spectrum Treatment

\section{Introduction}

The prevalence of a disease can be defined as the proportion of a population that has the disease at a specific level at one point in time [1]. In epidemiology, a patient has one or more diseases, while the rest of the population is healthy, therefore the patients determine the number of cases. In statistical analyses, sick or healthy individuals determine the number of cases. In epidemiology of dental caries, however, this principle has not been followed by the tra- 
ditional methods, as the statistical analysis is done with the tooth or surface units also considering their past caries experience. In other words, non-diseased units such as the restored ones can be included for the calculation of the prevalence [1].

An alternative to this traditional dental epidemiology approach is to use the Caries Assessment Spectrum Treatment (CAST) instrument. This method uses the individual unit to calculate the prevalence of caries without considering lost or restored teeth, which is more in agreement with the classical concept of prevalence [2]. CAST has been a method that has stood out in the epidemiology of caries and is simple, and easy to use. Its codes are arranged hierarchically, detecting the entire spectrum of caries lesions from those that involve enamel to those that lead to pulp exposure or caused fistula/abscess [3].

CAST can also measure the severity of caries in the individual or the population using a formula called F1. This formula was created and tested in 2017 [4] to replace the one that was initially conceived and published in the CAST manual (formula F) [5]. Having a tool that measures the severity of caries disease represents progress. The use of this formula establishes the severity of the disease in a determined population, which is important for the decisionmaking process in public health services, to improve communication between epidemiologists, and to facilitate the comparison between different populations.

In this study, the CAST severity score and the maximum CAST score per subject were calculated with and without the lost component (CAST 8 code) because the inclusion of the missing component has been questioned by some authors [6-9], mainly due to the impossibility of verifying whether the tooth was truly extracted by caries, which becomes more difficult in adults and can overestimate the presence of the caries disease.

Up to now, formula F1 has been tested in a group of children with mixed dentition, but has not yet been used in adults. As far as we know this is the first time that the formula is tested in this age group. The present article will test F1 and the maximum CAST score per subject including CAST code 8 (F1) or not (F1a) to verify its influence on the final severity score.

\section{Methods}

Students, employees, and their dependents, who were attending the Medical, Dental and Social Service (SMOS) at the State University of Bahia - UNEB, located in Salvador, Bahia, Brazil, during the period from September 6 to December 13, 2016, were examined. SMOS is a service for adults only, and the inclusion criterion has been in the care of the service during the period above.

The 260 individuals were examined after being adequately informed about the procedures of the study and having signed the informed consent form. The work was approved by the Research Ethics Committee of the Sciences Institute of the Federal University of Bahia under CAAE number 48500115.2.0000.5662.

Four trained and calibrated examiners, with the aid of three notetakers, applied CAST to the study population. The examinations were performed according to the recommendations of the CAST method's manual [5]. Re-examinations were performed in $10 \%$ of the sample to compare the intraexaminer and interexaminer reproducibility using Kendall's W Coefficient of Concordance [10].

The data were entered into Microsoft Excel (2007) and analyzed in R [11]. Further details on the calibration of examiners and data collection procedures are described in the article by Castro., et al. 2018 [12].

As recommended by the CAST Manual [5], the maximum score per subject was calculated. This indicator was also used without the lost component to verify the impact of missing teeth on the maximum CAST score per subject in adulthood.

With the CAST instrument, dentition and individuals are categorized into five stages [13]: the first stage is healthy, which includes the sound teeth or individual (CAST code 0 ) or the presence at least one tooth with sealant (CAST code 1) or when there is a filled tooth (CAST code 2). The second stage is called pre-morbidity when an enamel lesion is detected (CAST code 3).

The third stage is morbidity, when there is at least one tooth in the mouth with dentin carious lesion, cavitated or not [when there is a non-cavitated dentine carious lesion (CAST code 4) and if there is a cavitated dentine carious lesion (CAST code 5)]. A more severe degree of morbidity is the fourth stage (called serious morbidity) when there is at least one tooth with pulp involvement (CAST code 6) or abscess/fistula (CAST code 7).

The fifth stage, called mortality, refers to the existence of at least one tooth extracted due to caries (CAST code 8). This classification 
is based on the individual unit, but each subject may have one or 32 teeth attacked by the caries disease. To measure the different levels of caries that attacked the individual, it is necessary to use the CAST severity score through the dental unit.

The prevalence with the maximum CAST score per subject of caries includes only individuals who presented dentine carious lesions (CAST codes 4, 5, 6 and 7) [13], that is, only those subjects in the morbidity stage [5].

The CAST severity score [4] was previously applied in two different formulas (F and F1) to calculate the degree of caries severity, and it was concluded that F1 was the most appropriate one to establish the severity of caries disease in a child population. $\mathrm{F} 1=[0.25 *$ (CAST code 3$)+1 *($ CAST code 4$)+2 *($ CAST code 5$)+4 *($ CAST code 6$)+$ $5^{*}($ CAST code 7$)+6 *($ CAST code 8$\left.)\right]$.

The classification process of individuals in relation to the CAST severity score comprises three stages. In the first stage the highest CAST code observed on all surfaces of the examined tooth is determined; this code is used in the formula F1. In the second stage, after applying the formula and calculating the severity score of each individual, this group of people is classified into three levels according to disease severity [4] grouping the population into thirds: mild ( 0 - 33\%); moderate (33 - 66\%); and severe (> $66-100 \%)$. Finally, in the third stage, the highest and lowest CAST severity score is determined, with the minimum and maximum severity score values in each third, and through this the intervals of the severity score are calculated.

In adults, the lost tooth component may overestimate caries disease because many teeth may be lost for other reasons, such as periodontal disease, orthodontic treatment, fractures and abrasion [14]. Therefore, in this study, F1 was used with and without (F1a) the lost component.

\section{Results}

Most of the examined individuals were students (70.3\%) and female (74.2\%), with a mean age of 28 and a standard deviation (SD) of 10 years; aged 18 to 31 years old (73.0\%).

The median of the severity score when F1 was applied was 2.3, the interquartile range was 6.8 , the minimum value was 0 and the maximum was 108.0. The median of the severity score obtained using F1a was 1.0 , the interquartile range was 2.6 , the minimum value was 0 , and the maximum was 16.2 .
According to the CAST severity score after applying formula F1, $34.6 \%$ of the population were classified as presenting a mild condition, $31.2 \%$ as moderate, and $34.2 \%$ as severe. By applying F1a, the levels of dental caries changed: mild (35.0\%), moderate $(33.5 \%)$ or severe $(31.5 \%)$.

The classification of the severity score intervals calculated in the third stage using F1 were mild 0 - 0.75; moderate 0.76 - 5.24 and severe $>5.25$; while applying F1a the severity score intervals were mild 0 - 0.25; moderate $0.26-2.0$ and severe $>2.01$.

According to table 1, the percentage of subjects in the health stage increases from 20 to $25 \%$ if the lost teeth are not considered, $33.8 \%$ (with lost teeth) or $46.9 \%$ (without lost teeth) of the individuals examined had at least one enamel lesion as the worst condition observed so they are at the pre-morbidity stage, $13.1 \%$ (with lost teeth) and $23.1 \%$ (without lost teeth) had the worst condition observed dentin carious lesions without pulp involvement (morbidity stage) and 2.3\% (with lost teeth) and 5\% (without lost teeth) had at least one cavitated dentine carious lesion with pulp involvement at the serious morbidity stage and $30.8 \%$ (with lost teeth) had already had a tooth extracted due to caries (mortality stage).

\begin{tabular}{|c|c|c|c|c|c|}
\hline \multirow[t]{2}{*}{$\begin{array}{l}\text { Maximum score per } \\
\text { subject }\end{array}$} & \multicolumn{2}{|c|}{$\begin{array}{l}\text { CAST with } \\
\text { lost tooth }\end{array}$} & \multicolumn{2}{|c|}{$\begin{array}{l}\text { CAST with- } \\
\text { out lost } \\
\text { tooth }\end{array}$} & \multirow[t]{2}{*}{ Stage } \\
\hline & $\mathbf{N}$ & $\%$ & $\mathbf{N}$ & $\%$ & \\
\hline Sound (CAST codes $0,1,2$ ) & 52 & 20.0 & 65 & 25.0 & Healthy \\
\hline $\begin{array}{l}\text { Enamel lesion (CAST code } \\
\text { 3) }\end{array}$ & 88 & 33.8 & 122 & 46.9 & Pre-morbidity \\
\hline $\begin{array}{l}\text { Non-cavitated dentine } \\
\text { caries lesion (CAST code } \\
\text { 4) }\end{array}$ & 8 & 3.1 & 12 & 4.6 & \\
\hline $\begin{array}{l}\text { Cavitated dentine caries } \\
\text { lesion (CAST code } 5 \text { ) }\end{array}$ & 26 & 10.0 & 48 & 18.5 & Morbidity \\
\hline $\begin{array}{l}\text { Cavitated dentine caries } \\
\text { lesion with pulp involve- } \\
\text { ment (CAST code } 6 \text { ) }\end{array}$ & 6 & 2.3 & 13 & 5.0 & $\begin{array}{l}\text { Serious } \\
\text { morbidity }\end{array}$ \\
\hline $\begin{array}{l}\text { Lost due to caries (CAST } \\
\text { code } 8 \text { ) }\end{array}$ & 80 & 30.8 & & & Mortality \\
\hline
\end{tabular}

Table 1: Distribution of the maximum CAST score per subject with/ without lost teeth according to the different stages of severity. 
Table 2 shows the data from ten adults randomly selected (through a draw) with different distributions of teeth per CAST codes, with the stratification of individuals according to the CAST severity score with and without the lost component.

Subjects 03 and 04 had the same classification with or without the lost component because none of these patients lost their teeth throughout their life, but in the case of subjects $05,06,07$, and 08 , the classification changed from a minor to a major gravity stage when the lost teeth were removed.

Subject 08 was in the mild group without considering the lost component, but as he had lost eighteen teeth, he was reclassified to the severe group; however, there were no enamel or dentin lesions. In the case of patient 06 , he had no carious lesions and was correctly included in the mild group when lost teeth were not included, but although he had only one missing tooth, it was enough to reclassify the individual into the severe group when this tooth was included in the formula.

Subject 07 was also reclassified from the moderate to the severe group due to four extracted teeth, but this patient had only three teeth with enamel lesions, which does not characterize a severe degree of caries.

Patients 09 and 10 were classified at the moderate stage of caries severity with the missing component and in the severe stage without this component this can be explained because the missing component raises the prevalence of caries as a whole in the thirds that are used to establish the classification caries severity. The value found for

\begin{tabular}{lccccccccccccc}
\hline \multicolumn{1}{c}{$\begin{array}{l}\text { Patient } \\
\text { no. }\end{array}$} & d0 & d1 & d2 & d3 & $\mathbf{d 4}$ & $\mathbf{d 5}$ & $\mathbf{d 6}$ & $\mathbf{d 7}$ & $\mathbf{d 8}$ & Without lost tooth & With lost tooth \\
\hline 01 & 12 & 0 & 2 & 2 & 1 & 0 & 3 & 0 & 8 & 13.5 & Severe & 48 & Severe \\
02 & 21 & 0 & 3 & 3 & 0 & 1 & 0 & 0 & 0 & 2.75 & Severe & 2.75 & Moderate \\
03 & 16 & 0 & 8 & 4 & 0 & 0 & 0 & 0 & 0 & 1 & Moderate & 1 & Moderate \\
04 & 26 & 0 & 2 & 0 & 0 & 0 & 0 & 0 & 0 & 0 & Mild & 0 & Mild \\
05 & 5 & 0 & 5 & 3 & 1 & 0 & 0 & 0 & 14 & 1.75 & Moderate & 85.75 & Severe \\
06 & 21 & 0 & 6 & 0 & 0 & 0 & 0 & 0 & 1 & 0 & Mild & 6 & Severe \\
07 & 12 & 0 & 9 & 3 & 0 & 0 & 0 & 0 & 4 & 0.75 & Moderate & 24.75 & Severe \\
08 & 10 & 0 & 0 & 0 & 0 & 0 & 0 & 0 & 18 & 0 & Mild & 108 & Severe \\
09 & 19 & 0 & 7 & 1 & 0 & 0 & 1 & 0 & 0 & 4.25 & Severe & 4.25 & Moderate \\
10 & 16 & 0 & 4 & 7 & 0 & 1 & 0 & 0 & 0 & 3 & Severe & 3 & Moderate \\
\hline
\end{tabular}

Table 2: Teeth with caries assessment spectrum treatment codes and CAST severity score classification group of individuals with and without the lost component.

the CAST severity code for patient 09 was the same (5.25) with and without lost teeth, what changed was the third in which he was classified from severe to moderate.

\section{Discussion}

The study population was mostly composed of young adults $(73 \%$ between 18 and 30 years old), who presented a very low prevalence of caries lesions in dentin. This can be explained by the fact that these individuals had access to free dental care at the university's medical service; therefore, the teeth that needed to be restored or to be treated endodontically, had mostly received the treatment and the teeth that needed to be extracted were extracted. Taking this aspect into consideration, it can be stated the use of the CAST instrument is advantageous, as the changes promoted by the dental care implementation can be observed in the prevalence of the disease. 
The literature indicates that the CAST severity score is a useful tool in assisting policy-makers understanding how dental caries affects a certain population/individual, as it classifies them in relation to the severity of problem [15-17]. Moreover, measuring caries severity is seen as an advance in comparison to the traditional way of reporting dental caries, in that it makes it possible to identify more quickly whether the health of the population/individual is improving or getting worse [4].

According to the authors of the severity score [4], the inclusion of individuals into one of the three levels of dental caries severity (mild, moderate and severe) is limited by the prevalence and extent of caries in the population under study. In this study the use of the CAST severity score was very important to identify the third of the population that was most affected by the disease, and without the incorporation of the extracted component, this identification was made in a more precise way.

When the lost component was included in F1, the data became very heterogeneous because the difference between the interquartile range increased from 2.6 to 6.8 . Another problem, besides the fact that the distribution becomes much more asymmetrical when the lost component is included, is that this reflects much more the past disease of lost teeth that may have been extracted in childhood or adolescence than the current disease, which goes against the CAST philosophy of measuring the disease at the present time. Even considering that the loss of a tooth is something harmful, it is worth noting that in case of the adult population, subjects may not remember the cause of the tooth loss. Therefore, including this component in the CAST severity score is questionable.

The maximum CAST score per subject, proposed in the CAST Manual [5], is an important contribution of this method, as it allows easier communication with interlocutors from other areas when using the individual analysis unit, and the calculation of a population indicator of reversible caries injuries, as individuals with the worst oral health conditions are being cared for, the proportion of subjects with the worst scores will decrease.

With this tool, it was also possible to identify that the greatest need of the study group was health promotion and prevention actions, such as dietetic counselling, use of fluoride and sealants, reflected by the percentage of subjects presenting initial caries lesions
(33.8\% with missing teeth and $46.9 \%$ without missing teeth). This ability of the CAST instrument is in agreement with several other studies $[3,13,15]$.

However, our findings indicate that the inclusion of the missing component in adulthood can interfere with the maximum CAST score per subject, as shown in table 1 . This can be explained by the fact that the percentage of healthy individuals increased by removing the lost component by $5 \%$, and the percentage of subjects at the pre-morbidity stage (enamel lesions) increased by $13.1 \%$. For dentine caries lesions without pulp involvement (morbidity stage), this increase was of $12.7 \%$, and the number of individuals with at least one tooth with pulp involvement (serious morbidity stage) increased from 6 to 13 (2.7\%).

The inclusion of the lost component can hide the identification of people with cavitated dentine carious lesion with pulp involvement because the lost tooth is considered more serious. For example, according to table 2 with and without the lost component, patient 01 was classified as severe; however, when using the maximum CAST score per subject would move to the mortality stage, even though he presented 3 carious lesions reaching the pulp. By classifying this individual with code 8 , in the mortality group, it would not be possible to identify that this subject has an urgent need for dental care. By excluding the lost component, that need would be easily determined as the subject would be classified in the serious morbidity group (CAST code 6). Therefore, it can be stated that in adult populations with many teeth lost, it becomes difficult to identify a person who has an abscess or fistula as code 8 overlaps other codes; however, it is important to identify the individuals with such conditions so that the services can be organized to provide the appropriate treatment.

According to table 2 , some individuals $(05,06,07,08)$ were classified in a more severe stage in relation to caries disease due to the inclusion of the lost component, although only one of these patients had one caries lesion in dentin. In addition, in an adult population, the impact of including the missing component can be major because there are many teeth that were extracted due to periodontal disease or for economic reasons, mainly in underdeveloped or developing countries such as Brazil.

The lost component increases the severity level rating of the CAST score of severity, as in the example of subject 08 who had his score 
increased from mild to severe (0 to 108). On the other hand, the inclusion of this component can reclassify the individual from a more severe degree to a lighter degree of severity when the subject has no teeth extracted because the inclusion of missing teeth changed the cut-off values used to establish the classification of the severity of caries.

For example, patient 09 who had one tooth with a carious lesion that reached the pulp and only one enamel caries lesions with a CAST severity score value of 4.25 (the same value with and without the missing tooth because this subject had no missing teeth) and was reclassified from the severe group (severe $>2.25$ ) without the extracted component, to the moderate group with the inclusion of the lost component in calculating the severity score because with lost component the subject is classified in the group of severe when the score is higher than 5.25 .

The inclusion of the missing component is very important when examining children or adolescents because in this age group the lost component indicates the inability to reverse previous stages of carious disease ${ }^{4}$. Their teeth were probably lost due to caries, they were not lost due to periodontal disease or abrasion, for example. The tooth loss usually occurs in less time than in adulthood, which makes it easier for the child or parent to remember the reason for the extraction.

Further studies are needed using the CAST severity score to verify how this score behaves in other populations in other age groups with different levels of caries prevalence, but it is undoubtedly a promising and very useful tool that will help in the comparison of caries disease among individuals and populations.

\section{Conclusion}

The maximum CAST score per subject and the CAST severity score proved to be good tools to assess the severity and prevalence of caries in the study group; however, excluding the lost component resulted in better classification of individuals and made the values less heterogeneous.

\section{Conflict of Interest}

None declared.

\section{Bibliography}

1. Rothman KJ. "Precision and validity in epidemiologic studies". Modern epidemiology. Boston: Research Triangle Institute and Boston University (1998).
2. Frencken JE., et al. "The Caries Assessment Spectrum and Treatment (CAST) index: rational and development". International Dental Journal 61.3 (2011): 117-123.

3. Leal SC., et al. "Caries Assessment Spectrum and Treatment (CAST): A Novel Epidemiological Instrument". Caries Research 51.5 (2017): 500-506.

4. Ribeiro APD., et al. "Caries assessment spectrum treatment: the severity score". International Dental Journal 68.2 (2017): 84-90.

5. Leal S C FJ., et al. "Manual CAST: Caries Assessment and Treatment". In: Ipskamp Drukkers: Holanda (2015): 47.

6. Broadbent JM and Thomson WM. "For debate: problems with the DMF index pertinent to dental caries data analysis". Community Dentistry and Oral Epidemiology 33.6 (2005): 400-409.

7. Doyle J and Horowitz HS. "Influence of extracted teeth on DMF surface increments in clinical trials of caries preventives". Journal of Dental Research 49.6 (1970): 1417-1422.

8. Lawrence HP., et al. "Adjustment of the M-component of the DMFS index for prevalence studies of older adults". Community Dentistry and Oral Epidemiology 24.5 (1996): 322-331.

9. Larmas M. "Has dental caries prevalence some connection with caries index values in adults?" Caries Research 44.1 (2010): 81-84.

10. Kendall MG. "Rank correlation methods (1948).

11. R Core Team. "A language and environment for statistical computing. R Foundation for Statistical Computing, Vienna, Austria (2013).

12. Castro ALS., et al. "Comparison of caries lesion detection methods in epidemiological surveys: CAST, ICDAS and DMF". BMC Oral Health 18.1 (2018): 122.

13. Leal S., et al. "The Caries Assessment Spectrum and Treatment (CAST) Instrument". In: Detection and Assessment of Dental Caries (2019): 69-81.

14. Bikash D., et al. "To find out the causes of extraction of teeth in the patients coming to the department of oral and maxillofacial surgery at Kantipur dental college teaching hospital and research center from January 2014 to December 2018". Annals of Clinical and Medical Case Reports 2.2 (2019): 1-5. 
15. Maciel IP., et al. "Uso do instrumento CAST em levantamentos epidemiológicos: apresentação dos resultados em comparação ao critério da OMS". Ciencia and Saude Coletiva 24.9 (2019): 3529-3537.

16. Fisher J and Glick M. “Committee FWDFS, others. A New Model for Caries Classification and Management: The FDI World Dental Federation Caries Matrix. Elsevier (2012).

17. Mehta Abhishek. "Assessment of dental caries experience of an adult population using caries assessment spectrum and treatment index". SRM Journal of Research in Dental Sciences 8.1 (2017): 21.

Volume 5 Issue 9 September 2021

(C) All rights are reserved by Castro Ana Luiza Sarno., et al. 